\title{
Sole Excited-State InAs Quantum Dot Laser on Silicon With Strong Feedback Resistance
}

\author{
Jia-Jian Chen ${ }^{1}$, Zi-Hao Wang ${ }^{1,2}$, Wen-Qi Wei ${ }^{3}$, Ting Wang ${ }^{\star, 1,2,3}$ and Jian-Jun Zhang ${ }^{*, 1,2,3}$ \\ ${ }^{1}$ Beijing National Laboratory for Condensed Matter Physics, Institute of Physics, Chinese Academy of Sciences, Beijing, China, \\ ${ }^{2}$ Center of Materials Science and Optoelectronic Engineering, University of Chinese Academy of Sciences, Beijing, China, \\ ${ }^{3}$ Songshan Lake Materials Laboratory, Dongguan, China
}

OPEN ACCESS

Edited by:

Kar Wei Ng,

University of Macau, China

Reviewed by:

Sunil Singh Kushvaha,

National Physical Laboratory (CSIR),

India

Qiang Li,

Cardiff University, United Kingdom

*Correspondence:

Ting Wang

wangting@iphy.ac.cn

Jian-Jun Zhang

jzzhang@iphy.ac.cn

Specialty section:

This article was submitted to

Quantum Materials,

a section of the journal

Frontiers in Materials

Received: 31 December 2020

Accepted: 29 April 2021

Published: 07 June 2021

Citation:

Chen J-J, Wang Z-H, Wei W-Q,

Wang T and Zhang J-J (2021) Sole Excited-State InAs Quantum Dot Laser

on Silicon With Strong

Feedback Resistance.

Front. Mater. 8:648049.

doi: 10.3389/fmats.2021.648049
A feedback insensitive laser is a prerequisite for a desirable laser source for silicon photonic integration, as it is not possible to include an on-chip optical isolator. This work investigates the feedback insensitivity of an InAs/GaAs quantum dot laser epitaxially grown on an $\mathrm{Si}$ (001) substrate by operating in a sole excited state. The experimental results show that the sole excited-state lasing InAs quantum dot lasers on Si are less sensitive to external optical feedback than both Fabry-Perot and distributed-feedback quantum-well lasers. By comparing the laser behavior under different feedback levels, sole excited-state InAs quantum dot lasers on $\mathrm{Si}$ exhibit at least a $28 \mathrm{~dB}$ stronger feedback tolerance than quantum-well lasers. This result proposes a possible route for a high feedback insensitive laser as an on-chip light source towards Si waveguide integration with the absence of an optical isolator.

Keywords: quantum dot, external optical feedback, silicon photonic integration, distributed feedback laser, feedback sensitivity

\section{INTRODUCTION}

A photonic integrated circuit (PIC) on silicon is one of the most promising platforms for highdensity photonic integration (Dai et al., 2012; Komljenovic et al., 2018; Huang et al., 2020). Over the past decade, many methodologies have been implemented to integrate III-V materials onto silicon substrates, which take advantage of III-V materials as active devices, such as lasers and photodetectors (Zhang et al., 2019a; Feng et al., 2019; Malik et al., 2020; Grillot et al., 2020; Zhang et al., 2020; Wei et al., 2019; Wei et al., 2020a). But a major challenge impeding heterogeneous integration is the external optical feedback (EOF) induced between a laser and other photonic components or fiber connectors, which can lead the laser to operate in a chaotic state. Within optical modules, optical isolators are normally utilized to suppress the side effect of EOF (Bi et al., 2011; Hua et al., 2016), while increasing the overall cost and size of chip. As a result, lasers with lower sensitivity to EOF would be desirable for the high-density integration of PICs (Zhang et al., 2019b; Wei et al., 2020b).

It was proved that ground state (GS) InAs/GaAs quantum dot (QD) lasers can stably operate up to an optical feedback of $-7.4 \mathrm{~dB}$ (Duan et al., 2019). It is normally difficult to maintain sole GS in QD lasers at high injection current for higher output power as the stability of QD lasers is greatly affected by the mode competition between GS and excited state (ES) even when the GS still dominates. Especially at higher output power, the QD laser tends to operate in a dual state. Therefore, ES QD lasers become an optimal choice for an EOF insensitive optical source with high output power. The ES QD laser remains highly stable during operation at a high injection current while exhibiting a 
stronger feedback insensitivity than dual-state operation (Katsuaki et al., 2012). This paper investigates the feedback sensitivity of an InAs/GaAs QD laser epitaxially grown on $\mathrm{Si}$ (001) operating in a sole excited state. To note, the sole excitedstate lasers are highly stable during room-temperature operation but with a relatively short lifetime at the current stage.

The radio frequency (RF) spectra, optical spectra, and relative intensity noise (RIN) of commercial quantum-well (QW) lasers and InAs QD lasers on Si under varied EOF are investigated for direct comparison. To note, for commercial QW lasers, we examined both Fabry-Perot (FP) and distributed-feedback (DFB) lasers as reference samples. The experimental results are analyzed to achieve better insight into the understanding of sole excited-state operated InAs QD lasers under EOF.

In this paper, the feedback sensitivities of QW and QD FP lasers are firstly compared. The critical feedback level of an InAs QD FP laser on $\mathrm{Si}$ is $-7.8 \mathrm{~dB}$, which is significantly higher than the $-36 \mathrm{~dB}$ of the QW FP laser. The great advance of feedback insensitive QD lasers shows the significant potential of integrating on-chip lasers with silicon photonic components with the absence of optical isolators. Furthermore, in the case of QW lasers, the critical feedback level increases by $22 \mathrm{~dB}$ as the laser structure switches from the FP cavity to the DFB structure, which is attributed to the reflected light protection mechanism of DFB gratings. Therefore, we believe that an InAs QD DFB laser could potentially exhibit total feedback insensitivity properties with $100 \%$ back-reflection.

Analysis of feedback sensitivities of laser systems has already been undertaken, our observations on QD lasers and lasers with different structures subjected to a well-designed experimental circuit give an accurate measurement of the feedback sensitivities and are well supported by comprehensive characterizations. We feel that the quantitative comparison is particularly significant for particular optical system integrations.

\section{MATERIALS AND METHODS}

\section{Material Growth and Fabrication}

All material growth was conducted on an IV/III-V hybrid Molecular Beam Epitaxy (MBE) system. Figure 1A shows the schematic diagram of the InAs/GaAs QD laser grown on the $\mathrm{GaAs} / \mathrm{Si}$ (001) substrate. The GaAs/Si (001) platforms were achieved by growing III-V buffer layers on (111)-faceted silicon hollow substrates, which were obtained by homoepitaxy of a silicon buffer layer on "U"-shaped patterned Si (001) substrates. The AlGaAs/GaAs superlattices (SLs) and InGa(Al)As/GaAs quantum-well structures as dislocation filters (DFLs) were grown to flatten the GaAs surface and reduce the threading dislocation density (TDDs). The growth details and characterizations of GaAs/Si (001) platforms can be found in our previous work (Wang et al., 2011; Wei et al., 2018; Wei et al., 2019; Zhang et al., 2019a). As shown in Figure 1A, a $4 \mu \mathrm{m}$-thick InAs/GaAs QD laser structure mainly consists of topand bottom-AlGaAs cladding layers, GaAs contact layers, and a 7-layer InAs/GaAs dot-in-well (DWELL) active region. Each InAs/GaAs DWELL layer includes $8.1 \AA$ InAs QDs sandwiched by a $1.5 \mathrm{~nm}$ InGaAs wetting layer and a $4 \mathrm{~nm}$ InGaAs capping layer, separated by a $49 \mathrm{~nm}$ GaAs spacer layer. Figure 1B shows the photoluminescence (PL) spectrum
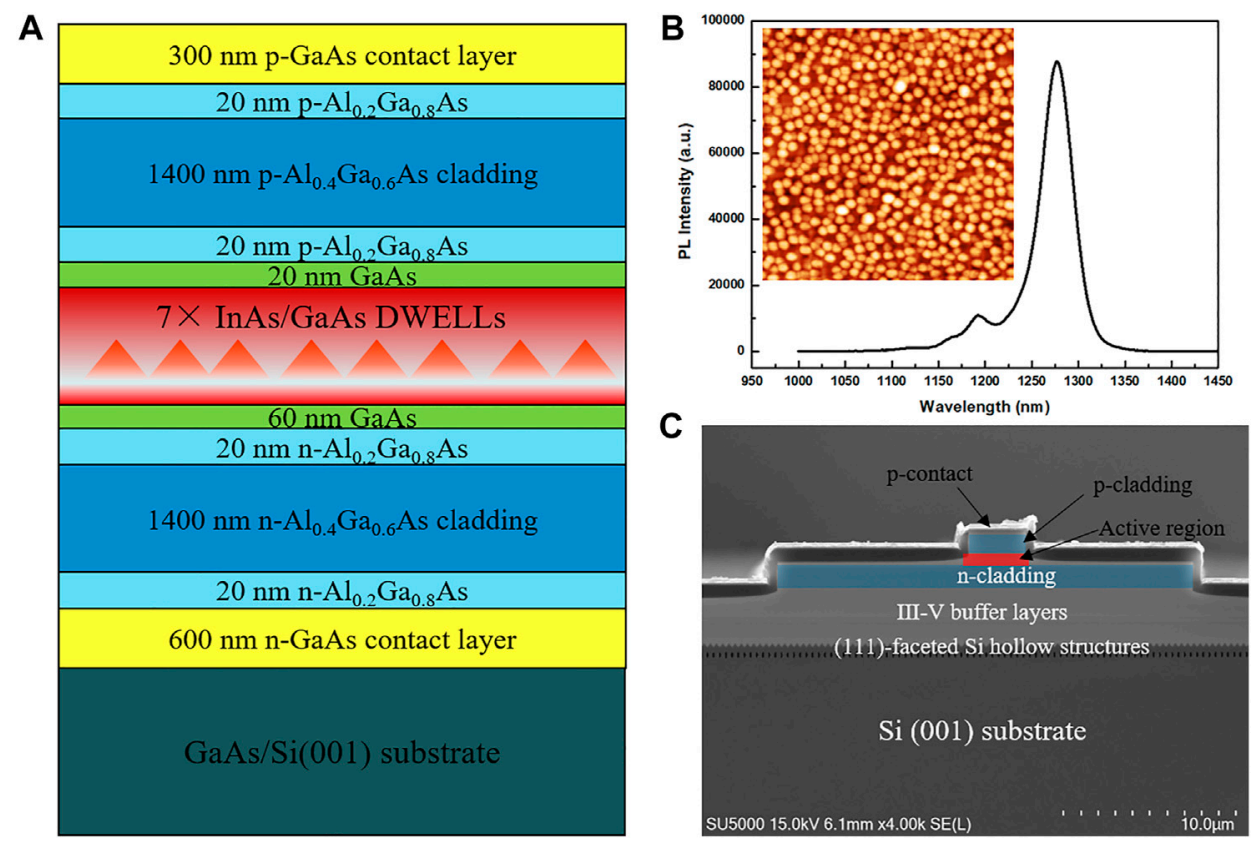

FIGURE 1 | (A) The schematic diagram of the whole InAs/GaAs QD laser structure on the GaAs/Si (001) substrate. (B) Room-temperature PL spectrum of a 7-layer InAs/GaAs DWELL structure grown on the GaAs/Si (001) substrate. Inset: $1 \times 1$ mm² AFM image of uncapped InAs/GaAs QDs on the GaAs/Si (001) substrate. (C) Colorenhanced cross-sectional SEM image of a fabricated ridge laser on the $\mathrm{Si}(001)$ substrate, with a ridge width of $4 \mu \mathrm{m}$. 
of the identical seven layers of InAs/GaAs DWELLs grown on the GaAs/Si (001) substrate. The PL spectrum presents a narrow emission peak of InAs/GaAs QDs on the Si (001) substrate, with a ground state peak wavelength of $1,276 \mathrm{~nm}$, a full width at half maximum (FWHM) of $30.4 \mathrm{meV}$, and an excited state peak wavelength of $1,190 \mathrm{~nm}$. The inset in Figure 1B displays the $1 \times 1 \mu \mathrm{m}^{2}$ AFM image of the surface InAs/GaAs QDs on the $\mathrm{GaAs} / \mathrm{Si}(001)$ substrate, which indicates the dot density of $5.12 \times$ $10^{10} \mathrm{~cm}^{-2}$.

Based on the materials prepared above, the narrow ridge FP lasers were fabricated with a ridge width of $4 \mu \mathrm{m}$ and a cavity length of $2 \mathrm{~mm}$. Figure $1 \mathrm{C}$ shows the color-enhanced crosssectional scanning electron microscopy (SEM) image of a $4 \mu \mathrm{m}$ wide ridge laser on the $\mathrm{Si}(001)$ substrate with "top-top" contacts. The as-cleaved ridge laser shows a clean and mirror-like facet as shown in the SEM image in Figure 1C.

\section{Experimental Set-Up}

The experimental arrangement is shown as below in Figure 2, the sole excited-state laser is continuous-wave (CW) current injected, and mounted on a thermoelectric cooler (TEC) to ensure stable temperature operation. The laser output is then coupled into lensed fiber. A 10/90 fiber coupler and an optical fiber circulator are implemented to couple the desirable output strength to the variable optical attenuator (VOA), in order to control the feedback ratio (which is defined as the feedback power to the total output power from the laser). In the experimental set-up described above, the feedback ratio can only be tuned from 0 to $19.95 \%$. In order to further measure the laser behavior at higher feedback levels, by connecting the 10/90 fiber coupler directly to a fiber back-reflector, the maximum measured feedback level can reach $50 \%$. An optical isolator is introduced at the detection end to eliminate additional unwanted feedback noise from the characterization equipment. The optical spectrum is measured by the optical spectrum analyzer (OSA) (Yokogawa AQ6370D), while a photodetector (Optilab PD-40M) with responsivity of 0.575 A/W@1,200 nm typ. and 0.640 A/W @ 1,300 nm typ. is used to capture the optical output to generate RF signal for electrical spectral analysis. The RF signal is amplified by the RF amplifier (Optilab MD50) with an electrical spectrum analyzer (ESA) (Agilent E4440A) set behind for frequency characterization.

In this work, a sole excited-state InAs QD laser on $\mathrm{Si}$ is operated under EOF ranging from -27 to $-3 \mathrm{~dB}$. An injection current of $130 \mathrm{~mA}$ and operating temperature of $15^{\circ} \mathrm{C}$ are selected here to ensure stable laser operation. Both the QW FP laser and QW DFB laser are tested under EOF ranging from -37 to $-7 \mathrm{~dB}$ with an injection current of 14 and $22 \mathrm{~mA}$, respectively.

\section{RESULTS AND DISCUSSION}

\section{Optical Spectral Analysis}

By implementing the experimental arrangement above, the evaluation of the optical spectral linewidth of a sole excitedstate QD laser on $\mathrm{Si}$ is achieved as shown in Figure 3. In Figure 3A, significant laser linewidth broadening of the InAs QD laser on Si occurs at an EOF of $-7.8 \mathrm{~dB}$, which leads to a clear coherence collapse. Besides, the isolated linewidths under EOF both below and above the critical feedback level are investigated to further confirm the evolution of optical spectra. Figure 3D gives a clear demonstration of change of linewidth under different EOF. This broadening represents the start of chaotic state in the QD laser as shown in Figure 3A.

For comparison, both the commercial QW FP laser and DFB laser are characterized here for optical spectral analysis. The optical spectral mapping of the QW FP laser in Figure 3B shows continuous broadening under an EOF above $-36 \mathrm{~dB}$, which is confirmed by the evolution of the linewidth in Figure 3E. Simultaneously, we measured the optical spectral evolution of the QW DFB laser as shown in Figures 3C,F. In Figure 3C, the optical spectrum shows coherence collapse at an EOF of $-14 \mathrm{~dB}$, which is confirmed by the demonstration of linewidth evolution in Figure 3F. This result indicates that the QW DFB laser exhibits a stronger resistance against feedback with an increased critical feedback level of $22 \mathrm{~dB}$ over the FP structure with identical QW structures. The observed relatively high critical feedback level of the QW DBF laser benefits from the

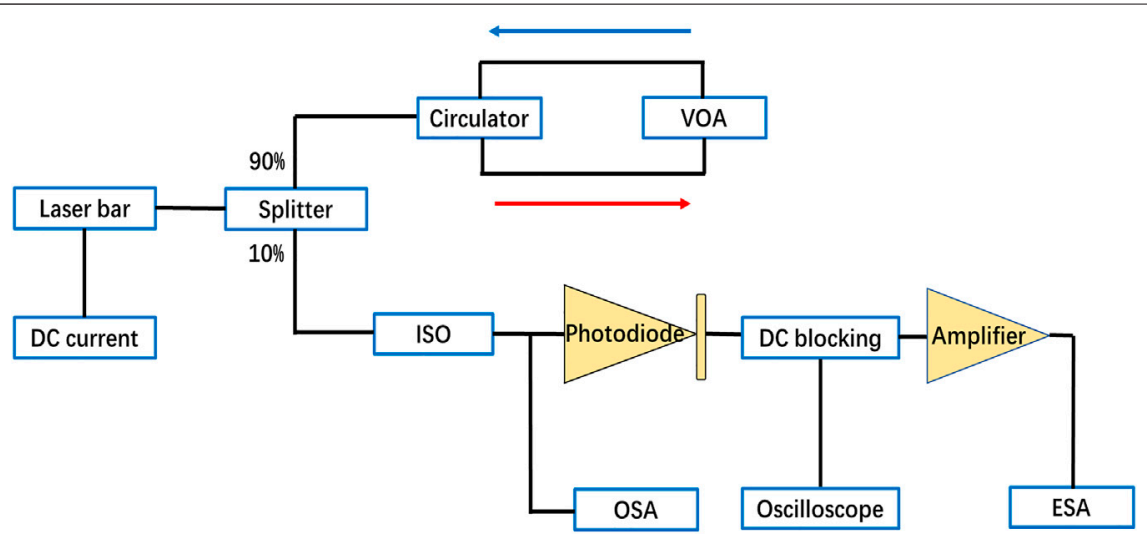

FIGURE 2 | Experimental arrangement for the measurements of optical and RF spectra of QD lasers under different optical feedback. VOA, variable optical attenuator; ISO, optical isolator; OSA, optical spectrum analyzer; ESA, electrical spectrum analyzer. 
reduced feedback strength inside the laser cavity due to the DFB gratings. The overall optical spectral evolution analysis among all three types of lasers shows that the sole ES QD laser exhibits superior performance over the QW laser with an improved critical feedback level of $28.2 \mathrm{~dB}$ in the identical FP structure.

\section{Radio Frequency Spectral Analysis}

In order to verify the feedback resistance results extracted from optical spectral evolution, feedback-dependent measurements in the electrical frequency domain are also performed here. However, the critical feedback levels in ESA could be different from those in OSA due to the coupling variance during testing, therefore the values from optical spectra in Figure 3 will be adopted as the credible result for our experimental set-up.

The resonance frequency of FP modes can be calculated by Eq. 1.

$$
f=\frac{c}{2 n_{e f f} L}
$$

where $c$ is light speed in vacuum; $n_{\text {eff }}$ is the effective index of the material; and $\mathrm{L}$ is the distance from the laser to the external feedback facet.

The length of the total optical path is defined as the round trip between the front laser facet and the VOA, which is $8 \mathrm{~m}$ in our experimental set-up. As a result, the fundamental FP resonance frequency of the external circuit is $12.8 \mathrm{MHz}$ while the frequency resolution of ESA in this experiment is $16.7 \mathrm{MHz}$. This explains the absence of fundamental FP resonance peaks in the following RF spectra and RIN.

Quasiperiodic peaks are observed between 0.5 and $3 \mathrm{GHz}$ in Figure 4A when EOF reaches $-15 \mathrm{~dB}$, but they show little impact on the coherent lasing of the QD laser according to Figures 3A,D, so they should represent quasiperiodic relaxation oscillation (RO). Under an increased EOF of $-7 \mathrm{~dB}$, chaotic oscillation (CO) occurs over a range of $3-6.5 \mathrm{GHz}$, which represents the coherence collapse of the laser. The difference in critical levels acquired from OSA and ESA could come from the variance in coupling efficiency during tests.
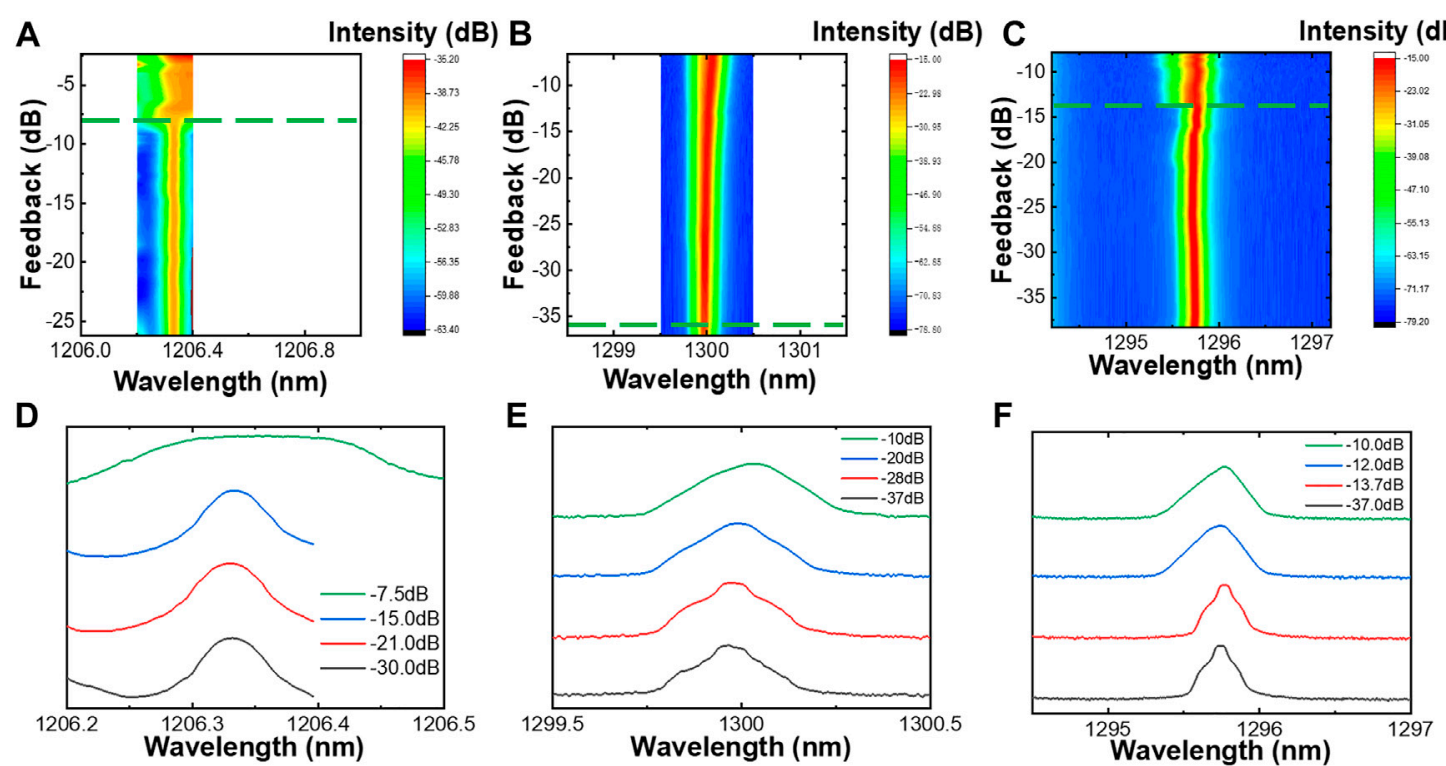

FIGURE 3 | The evolution of optical spectra of (A,D) the QD FP laser; (B,E) QW FP laser; and (C,F) QW DFB laser.
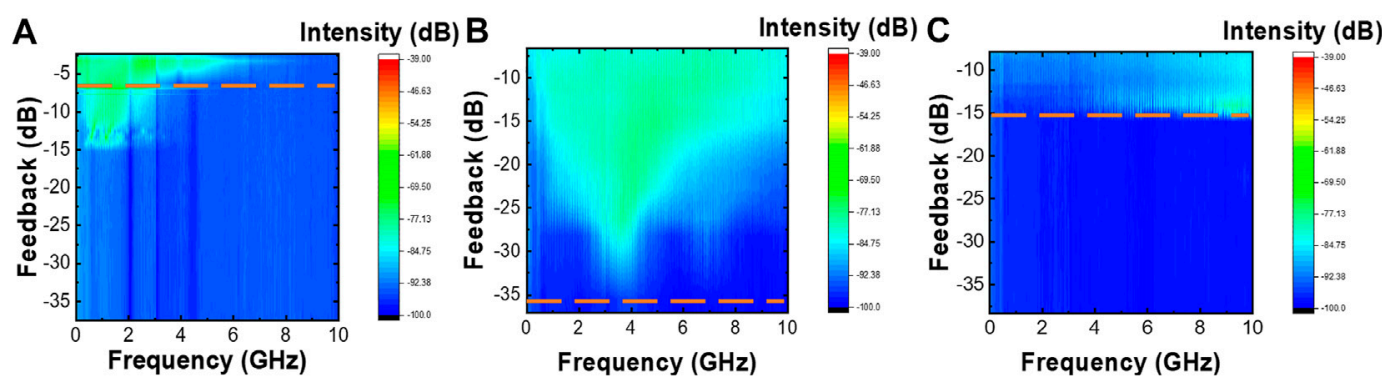

FIGURE 4 | The evolution of electrical spectra of (A) the QD FP laser; (B) QW FP laser; and (C) QW DFB laser. 
In the case of the QW FP laser, the RF spectrum shows initial $\mathrm{CO}$ peaks ranging from 3 to $4 \mathrm{GHz}$ under an EOF of $-36 \mathrm{~dB}$ as shown in Figure 4B, followed by full chaotic operation, which is in line with the optical linewidth broadening previously observed. This result confirms that the coherence collapse in the QW FP laser happens at an EOF of $-36 \mathrm{~dB}$. The critical feedback level difference acquired from Figures 4A,B strongly supports the superior feedback insensitivity performance of QD materials.

Figure 4C shows the measured ESA spectral mapping of the QW DFB laser with increasing EOF. An abrupt increase in chaotic states occurs under an EOF of $-15 \mathrm{~dB}$. The comparison between Figures 4B,C suggests that the critical feedback level in the DFB QW laser is at least $21 \mathrm{~dB}$ higher than that of the QW FP laser, due to the grating structure.

\section{Relative Intensity Noise Measurements}

We also performed relative intensity noise (RIN) measurements for three different laser structures. The RIN of an operating laser can be described by Eq. 2. (Zhou et al., 2017):

$$
R I N(f)=10 \log _{10}\left[\frac{\frac{N_{T}-N_{t h}}{R B W \times G}-N_{q}}{P_{D C}}\right](d B / H z)
$$

where $N_{T}$ is the total noise in the ESA spectrum; $N_{t h}$ is the thermal noise from the experimental set-up; $\mathrm{RBW}=\mathrm{VBW}=200 \mathrm{KHz}$; $\mathrm{G}$, the gain of the RF amplifier is $30 \mathrm{~dB} ; N_{q}$ is the shot noise from the photodetector, defined as $N_{q}=2 q I_{D C} R_{L}$, where $\mathrm{q}$ is the elementary charge $I_{D C}$ is the DC current extracted from DC blocking, and $R_{L}$ is the resistance of ESA; and $P_{D C}$ is the DC power.

Usually, coherence collapse is defined as a collective phenomenon of the broadening of the laser spectrum with significant increases, such as an abrupt increase of RIN and frequency fluctuation (Cohen and Lenstra., 1991; Lenstra et al., 2019). Here in this work, we analyze the RIN spectra with different EOF below and above the critical feedback level to understand the dynamics of semiconductor lasers with feedback.

In Figure 5A, the QD laser shows relatively flat RIN spectra until $-15 \mathrm{~dB} E O F$, where the quasiperiodic RO frequency peaks arise, which however has no influence on the coherent operation of the laser as the optical linewidth remains the same as in Figure 3D. By further increasing the EOF level towards $-7.5 \mathrm{~dB}$, a broad electrical signal noise peak appears, which represents the coherence collapse of the QD laser. This behavior suggests that the electrical noise peaks over the frequency range above $3 \mathrm{GHz}$ represents the dominant noise frequency that influences optical feedback sensitivity.

For the QW FP laser, even under a weak EOF of $-37 \mathrm{~dB}$, multiple frequency noise peaks already exist in the RIN diagram in Figure 5B. The periodic peaks could be the high-order harmonics of the fundamental RO frequency, which are caused by the intracavity resonance, and thus the coherence collapse follows in the RF spectrum and RIN diagram (Lin et al., 2018). With increasing EOF levels, the noise level continuously grows, which leads to significant optical spectral broadening in Figures 3B,E.

The initial peak of RIN shows up at an EOF of $-13.7 \mathrm{~dB}$, followed by broadening in the QW DFB laser, which is shown in Figure 5C. This agrees with the result from optical linewidth evolution in Figures 3C,F and the electrical spectrum in Figure 4C.

From electrical spectral analysis, including Figures $4 A, B, 5 A, B$, the QD laser and QW laser clearly undergo different routes into chaotic states. The QD laser shows quasiperiodic oscillation of RO frequency before coherence collapse in the range of EOF from $-15 \mathrm{~dB}$ to $-7.5 \mathrm{~dB}$. In comparison, the QW laser goes through a high-order harmonics RO process before moving into chaotic states. Different chaotic routes in $\mathrm{QD}$ lasers come from a unique quantum dots structure with higher confinement and thus reduces high-order harmonics behavior in the FP structure. The discrete energy state of QD lasers causes a lower enhancement factor and higher damping factor which are also well-accepted explanations for the higher feedback insensitivity of QD lasers over QW lasers (Duan et al., 2018). A lower enhancement factor suppresses the noise caused by EOF which otherwise can be amplified in the gain medium towards a chaotic operation (Lenstra et al., 2019).

The superior performance of the DFB structure lies in the distributed-feedback grating structure which can reduce the EOF strength entering the resonant cavity from the external circuits, while the FP laser will be strongly affected by the entered optical feedback. The suppression of high-order harmonics in DFB, which reduces the multimode competition in lasers, is also a main mechanism for higher feedback resistance.

The results in this paper analyzed the critical EOF level in different materials and structures. The high feedback insensitivity of the ES QD laser and the feedback noise suppression capability in the DFB structure are confirmed. We believe these results make it possible to fabricate better EOF insensitive QD DFB lasers, which can potentially achieve stable coherent operation with $100 \%$ EOF for future photonic integrations.
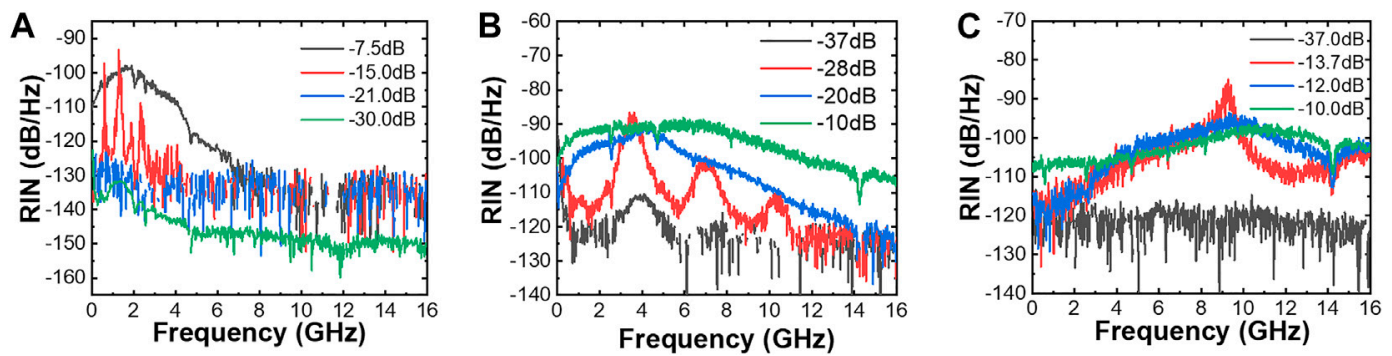

FIGURE 5 | The evolution of RIN of (A) the QD FP laser; (B) QW FP laser; and (C) QW DFB laser. 


\section{CONCLUSION}

To summarize, we have investigated the feedback sensitivity of an ES InAs/GaAs QD laser on silicon in comparison with commercial QW lasers. The analyzed optical spectral mapping, electrical spectral mapping, and RIN spectra confirm the superior performance of the sole ES InAs QD laser on silicon over QW lasers, which benefits from the absence of mode competition. The measured maximum optical feedback tolerance is $-7.8 \mathrm{~dB}$ for the sole ES InAs QD laser on silicon, which is almost $28 \mathrm{~dB}$ higher than the QW laser. In addition, the relatively better feedback insensitivity of QW DFB lasers suggests that the DFB structure utilizing QD material is potentially resistant to $100 \%$ of feedback, which could be promising for future on-chip photonic integration, where there are no on-chip optical isolators. Although the excited-state QD laser exhibited strong feedback resistance, the relatively shorter operating wavelength and lossy cavity will be carefully redesigned for future applications.

\section{DATA AVAILABILITY STATEMENT}

The original contributions presented in the study are included in the article/Supplementary Material, further inquiries can be directed to the corresponding authors.

\section{REFERENCES}

Bi, L., Hu, J., Jiang, P., Kim, D. H., Dionne, G. F., Kimerling, L. C., et al. (2011). Onchip Optical Isolation in Monolithically Integrated Non-reciprocal Optical Resonators. Nat. Photon. 5, 758-762. doi:10.1038/NPHOTON.2011.270

Cohen, J. S., and Lenstra, D. (1991). The Critical Amount of Optical Feedback, for Coherence Collapse in Semiconductor Lasers. IEEE J. Quan. Electron. 27 (1), 10-12. doi:10.1109/3.73535

Dai, D., Bauters, J., and Bowers, J. E. (2012). Passive Technologies for Future LargeScale Photonic Integrated Circuits on Silicon: Polarization Handling, Light Nonreciprocity and Loss Reduction. Light Sci. Appl. 1, e1. doi:10.1038/lsa.2012.1

Duan, J., Huang, H., Dong, B., Jung, D., Norman, J. C., Bowers, J. E., et al. (2019). 1.3- $\mu \mathrm{m}$ Reflection Insensitive InAs/GaAs Quantum Dot Lasers Directly Grown on Silicon. IEEE Photon. Technol. Lett. 31 (5), 345-348. doi:10.1109/ LPT.2019.2895049

Duan, J., Huang, H., Jung, D., Zhang, Z., Norman, J., Bowers, J. E., et al. (2018). Semiconductor Quantum Dot Lasers Epitaxially Grown on Silicon with Low Linewidth Enhancement Factor. Appl. Phys. Lett. 112, 251111-251125. doi:10. 1063/1.5025879

Feng, Q., Wei, W., Zhang, B., Wang, H., Wang, J., Cong, H., et al. (2019). O-band and C/L-Band III-V Quantum Dot Lasers Monolithically Grown on Ge and Si Substrate. Appl. Sci. 9, 385. doi:10.3390/app9030385

Grillot, F., Duan, J., Dong, B., Huang, H., Liu, S., Chow, W. W., et al. (2020). "Quantum Dot Lasers Based Photonics Integrated Circuits," in 2020 IEEE Photonics Conference (IPC), Vancouver, BC, Canada, September 28-October 1, 2020. doi:10.1109/IPC47351.2020.9252213

Hua, S., Wen, J., Jiang, X., Hua, Q., Jiang, L., and Xiao, M. (2016). Demonstration of a Chip-Based Optical Isolator with Parametric Amplification. Nat. Commun. 7, 13657. doi:10.1038/ncomms13657

Huang, H., Duan, J., Dong, B., Norman, J., Jung, D., Bowers, J. E., et al. (2020). Epitaxial Quantum Dot Lasers on Silicon with High thermal Stability and strong Resistance to Optical Feedback. APL Photon. 5, 016103. doi:10.1063/1. 5120029

Katsuaki, T., Watanabe, K., and Arakawa, Y. (2012). III-V/Si Hybrid Photonic Devices by Direct Fusion Bonding. Sci. Rep. 2, 349. doi:10.1038/srep00349

\section{AUTHOR CONTRIBUTIONS}

J-JC wrote the manuscript. All authors discussed the results and contributed the final manuscript.

\section{FUNDING}

This research was funded by Guangdong Key-Area Research and Development Program of Guangdong Province (No. 2019B121204003), National Natural Science Foundation of China (Grant Nos. 61975230, 61635011, 61804177, 62005308, and 11804382), National Key Research and Development Program of China (No. 2018YFB2200104), Beijing Municipal Science and Technology Commission (No. Z191100004819010), and the Key Research Program of Frontier Sciences, CAS (Grant No. QYZDB-SSW-JSC009). TW was supported by the Youth Innovation Promotion Association of CAS (No. 2018011).

\section{ACKNOWLEDGMENTS}

The authors would like to thank Jingjing Guo, Mingchen Guo, and Kaiyue Hou for their constructive contributions in material growth and device fabrications.

Komljenovic, T., Huang, D., Pintus, P., Tran, M. A., Davenport, M. L., and Bowers, J. E. (2018). Photonic Integrated Circuits Using Heterogeneous Integration on Silicon. Proc. IEEE. 106 (12), 2246-2257. doi:10.1109/JPROC.2018.2864668

Lenstra, D., Van Schaijk, T. T. M., and Williams, K. A. (2019). Toward a FeedbackInsensitive Semiconductor Laser. IEEE J. Select. Top. Quan. Electron. 25 (6), 1-13. doi:10.1109/JSTQE.2019.2924139

Lin, L.-C., Chen, C.-Y., Huang, H., Arsenijević, D., Bimberg, D., Grillot, F., et al. (2018). Comparison of Optical Feedback Dynamics of InAs/GaAs QuantumDot Lasers Emitting Solely on Ground or Excited States. Opt. Lett. 43 (2), 210-213. doi:10.1364/OL.43.000210

Malik, A., Guo, J., Tran, M. A., Kurczveil, A. G., Liang, D., Bowers, J. E., et al. (2020). Widely Tunable, Heterogeneously Integrated Quantum-Dot O-Band Lasers on Silicon. Photon. Res. 8, 1551. doi:10.1364/PRJ.394726

Wang, T., Liu, H., Lee, A., Pozzi, F., and Seeds, A. (2011). 13- $\mu \mathrm{m}$ InAs/GaAs Quantum-Dot Lasers Monolithically Grown on Si Substrates. Opt. Express. 19, 11381-11386. doi:10.1364/oe.19.011381

Wei, W.-Q., Wang, J.-H., Zhang, B., Zhang, J.-Y., Wang, H.-L., Feng, Q., et al. (2018). InAs QDs on (111)-faceted Si (001) Hollow Substrates with strong Emission at $1300 \mathrm{Nm}$ and $1550 \mathrm{Nm}$. Appl. Phys. Lett. 113 (5), 053107, doi:10. $1063 / 1.5043169$

Wei, W.-Q., Feng, Q., Guo, J.-J., Guo, M.-C., Wang, J.-H., Wang, Z.-H., et al. (2020a). InAs/GaAs Quantum Dot Narrow ridge Lasers Epitaxially Grown on SOI Substrates for Silicon Photonic Integration. Opt. Express. 28 (18), 26555. doi:10.1364/oe.402174

Wei, W.-Q., Wang, J.-H., Zhang, J.-Y., Feng, Q., Wang, Z., Xu, H.-X., et al. (2020b). A CMOS Compatible Si Template with (111) Facets for Direct Epitaxial Growth of III-V Materials. Chin. Phys. Lett. 37 (2), 024203. doi:10.1088/0256-307X/37/ $2 / 024203$

Wei, W., Feng, Q., Wang, Z., Wang, T., and Zhang, J. (2019). Perspective: Optically-Pumped III-V Quantum Dot Microcavity Lasers via CMOS Compatible Patterned Si (001) Substrates. J. Semicond. 40 (10), 101303. doi:10.1088/1674-4926/40/10/101303

Zhang, B., Wei, W.-Q., Wang, J.-H., Wang, H.-L., Zhao, Z., Liu, L., et al. (2019a). O-band InAs/GaAs Quantum-Dot Microcavity Laser on Si (001) Hollow Substrate by Iin-Ssitu Hybrid Epitaxy. AIP Adv. 9 (1), 015331. doi:10.1063/ 1.5065527 
Zhang, B., Wei, W.-Q., Wang, J.-H., Zhang, J.-Y., Cong, H., Feng, Q., et al. (2019b). $1310 \mathrm{Nm}$ InAs Quantum-Dot Microdisk Lasers on SOI by Hybrid Epitaxy. Opt. Express. 27, 19348. doi:10.1364/oe.27.019348

Zhang, J.-Y., Wei, W.-Q., Wang, J.-H., Cong, H., Feng, Q., Wang, Z.-H., et al. (2020). Epitaxial Growth of InAs/GaAs Quantum Dots on $\{113\}$-faceted Ge/Si (001) Hollow Substrate. Opt. Mater. Express. 10 (4), 1045. doi:10.1364/ome. 383742

Zhou, Y.-G., Zhou, C., Cao, C.-F., Du, J.-B., Gong, Q., and Wang, C. (2017). Relative Intensity Noise of InAs Quantum Dot Lasers Epitaxially Grown on Ge. Opt. Express. 25 (23), 28817. doi:10.1364/oe.25.028817
Conflict of Interest: The authors declare that the research was conducted in the absence of any commercial or financial relationships that could be construed as a potential conflict of interest.

Copyright (c) 2021 Chen, Wang, Wei, Wang and Zhang. This is an open-access article distributed under the terms of the Creative Commons Attribution License (CC BY). The use, distribution or reproduction in other forums is permitted, provided the original author(s) and the copyright owner(s) are credited and that the original publication in this journal is cited, in accordance with accepted academic practice. No use, distribution or reproduction is permitted which does not comply with these terms. 